\title{
Interactions of arbuscular mycorrhizal and endophytic fungi improve seedling survival and growth in post-mining waste
}

\author{
Katarzyna Wężowicz ${ }^{1}$ - Piotr Rozpądek ${ }^{2}$ - Katarzyna Turnau ${ }^{1}$
}

Received: 18 November 2016/Accepted: 10 March 2017 / Published online: 20 March 2017

(C) The Author(s) 2017. This article is published with open access at Springerlink.com

\begin{abstract}
The impact of fungal endophytes and the modulating role of arbuscular mycorrhizal fungi (AMF) on the vitality of Verbascum lychnitis, grown in the laboratory in a substratum from a post-mining waste dump was investigated. We report that inoculation with a single endophyte negatively affected the survival rate and biomass production of most of the plant-endophyte consortia examined. The introduction of arbuscular mycorrhiza fungi into this setup (dual inoculation) had a beneficial effect on both biomass yield and survivability. V. lychnitis co-inoculated with AMF and Cochliobolus sativus, Diaporthe sp., and Phoma exigua var. exigua yielded the highest biomass, exceeding the growth rate of both noninoculated and AMF plants. AMF significantly improved the photosynthesis rates of the plant-endophyte consortia, which were negatively affected by inoculation with single endophytes. The abundance of PsbC, a photosystem II core protein previously shown to be upregulated in plants colonized by Epichloe typhina, exhibited a significant increase when the negative effect of the fungal endophyte was attenuated by AMF.
\end{abstract}

Keywords AMF · Fungal endophytes - Verbascum lychnitis . Toxic metals $\cdot$ Photosynthesis efficiency

Electronic supplementary material The online version of this article (doi:10.1007/s00572-017-0768-x) contains supplementary material, which is available to authorized users.

Piotr Rozpądek

piotr.rozpadek@uj.edu.pl

1 Institute of Environmental Sciences, Jagiellonian University, Gronostajowa 7, 30-387 Kraków, Poland

2 Malopolska Centre of Biotechnology, Jagiellonian University, Gronostajowa 7A, 30-387 Kraków, Poland

\section{Introduction}

Plants, in their natural environments, are a refuge for numerous organisms ranging from small mammals, through invertebrates and fungi, to unicellular bacteria (Beckett et al. 1992; Lindow and Brandl 2003). From the functional standpoint, a single plant can be referred to as a specific "microcosmos," where structure and functions are organized by a dense net of multidimensional interactions between the plant and its inhabitants, as well as by interactions between the organisms colonizing the plant (Van der Putten et al. 2001; Singh et al. 2004; Felton and Tumlinson 2008; Aly et al. 2011). The symbiosis of plants has been the subject of a multitude of studies. Microorganisms, fungi, and bacteria take central positions in this field of symbiosis due to their impacts on the host's biology and potential application.

Microorganisms inhabiting the plant differ in their trophic strategy ranging from pathogens and parasites to mutualists (Redman et al. 2001). Additionally, the character of the relationship of a specific microorganism and its host plant is variable and may change over time. In many cases, abiotic and biotic constraints such as resource availability, herbivores, factors limiting carbon assimilation, and stage of ontogeny among others may elicit a switch from mutualism to parasitism and vice-versa (Kiers et al. 2010). In plant symbiotic interactions, the microorganism functions as an additional sink for carbohyrates. In instances where the costs of maintaining a fungal partner do not exceed benefits, the plant acts as the most effective provider to the fungus. However, under environmental challenges, a trade-off in resource allocation may limit carbon flow from the plant to its symbiotic partner and disturb the mutualistic equilibrum (Ahlholm et al. 2002; Bever et al. 2009). This phenomenon, described for symbiotic fungi, is referred to as the endophyte-parasite continuum (Schulz and Boyle 2005; Mandyam et al. 2013; Reininger 
and Schlegel 2016). Additionally, the presence of fungi and bacteria with pest control capabilities may influence the lifestyle of co-inhabitants. This ability of symbiotic microorganisms may be exploited as an environmentally friendly alternative for traditional pest control in agriculture (Doan et al. 2008; Jäschke et al. 2010).

The growing deposition of toxic metals in the environment severely restricts plant growth. This has serious economic and social implications, due to yield losses in agriculture, contamination of water reservoirs, and increased incidence of diseases. A promising method for restoring areas polluted by industrial activities is phytoremediation. Phytoremediation utilizes the ability of plants to remove and/or immobilize a wide range of organic and inorganic pollutants, including toxic metals deposited in the substratum (Turnau et al. 2006).

The remedial capacity of plants can be greatly improved by microorganisms such as bacteria and fungi. There are two major classes of ubiquitous fungal symbionts associated with plants in terrestrial ecosystems: arbuscular mycorrhizal fungi (AMF) and fungal endophytes. The roots of more than $80 \%$ of plants on land are colonized by AMF (Smith and Read 2008). The benefits related to mycorrhization include increased root absorption area and hence increased uptake of nutrients (Smith and Read 1997a, 1997b), a better tolerance to water stress (Porcel and RuizLozano 2004), high salinity (Daei et al. 2009), pathogens (Cordier et al. 1998), and toxic metals (Turnau et al. 2006). Endophytes are commonly isolated from roots and shoots of plants (Stone et al. 2000; Zhang et al. 2006; Wężowicz et al. 2014). Their presence within host tissues may promote plant growth, nutrient acquisition, and confer tolerance against abiotic and biotic stresses (Redman et al. 2002; Lewis 2004; Schulz and Boyle 2005; Waller et al. 2005; Hiruma et al. 2016), including toxic metal tolerance (Deng et al. 2011; Li et al. 2011a; Li et al. 2011b; Li et al. 2012). Fungal endophytes have been shown to possess a remarkable ability to accumulate toxic metals: the DSE (dark septate endophyte) Exophiala pisciphila (H93) from the roots of Urundinella bengalensis accumulated over 5\% Cd in its dry mass (Zhang et al. 2008). Interestingly, DSEs are ubiquitous and colonize roots of plants growing in extremely unfavorable, post-industrial environments. Furthermore, their colonization increases with the growing quantity of toxic metals in the substratum (Regvar et al. 2010; Shen et al. 2015; Zhao et al. 2015).

Excess of toxic metals alters photosynthesis functions at various levels of organization. For instance, disturbances in chlorophyll biosynthesis and thylakoid electron transport are induced in the presence of $\mathrm{Pb}, \mathrm{Zn}$, and $\mathrm{Cd}$ (Tripathy and Mohanty 1980; Sheoran et al. 1990; Stefanov et al. 1995; Vodnik et al. 1999; Villiers et al. 2011). As a result, plant growth and development is severely impaired. Symbiosis with fungi was reported to attenuate the effects of toxic metals stress. Inoculation with fungal endophytes increased photosynthetic pigment abundance and enhanced water use efficiency, promoting photosynthesis and rice growth in $\mathrm{Pb}$ stress conditions (Li et al. 2012). Mycorrhizal rice showed higher chlorophyll content and photosynthetic efficiency in the presence of As, in comparison to non-mycorrhizal plants (de Andrade Lopez et al. 2015). AMF were shown to upregulate detoxification mechanisms including the antioxidant system. Efficient ROS (reactive oxygen species) scavenging is crucial to maintaining proper function of electron transport and subsequent ATP and NADPH production in the photosynthetic electron transport chain. Improved photosynthetic efficiency was attributed not only to the protective role of the fungi, but also, according to Rozpadek et al. (2015), to symbiont-related alterations in the composition, i.e. the abundance of specific photosystem proteins, including PsbC and Lhcb3 that may positively impact the function of the photosynthesis apparatus.

So far, AMF-plant or endophyte-plant interactions were studied in single plant-fungus models. The few coinoculation studies, where plants were inoculated by more than one group of fungi, were performed using grasses as the host plants. The results of these experiments are not ambiguous, showing that the microorganisms inhabiting plant hosts positively influenced plant growth (Mack and Rudgers 2008; Scervino et al. 2009; Larimer et al. 2012).

AMF have been described as important factors in remediation strategies (Leyval et al. 1997; Turnau et al. 2005). The role of endophytes in this process also has been well demonstrated (Weyens et al. 2009 and publications cited there; Soleimani et al. 2010; Li et al. 2012 and publications cited there). Nevertheless, the majority of research related to the use of endophytes in phytoremediation comes from studies of bacterial endophytes. Endophytic fungi are yet to receive the appropriate attention from the scientific community. In a previously published article, the diversity of fungal endophytes from Verbascum lychnitis, a biennial herb from the Scrophulariaceae family, inhabiting post-industrial wastelands in South Poland was shown (Wężowicz et al. 2014). The aim of this study was to evaluate the growth response, photosynthesis performance, and possible alterations in the composition of selected photosynthetically active pigments and structural proteins of $V$. lychnitis inoculated with selected endophytic fungi and co-inoculated with endophytes and AMF. The plants were cultivated in the substratum of the mine dump "Chrzanów" under laboratory conditions. This permitted selection of fungal endophytes with the highest potential for utilization in phytoremediation. We predicted that the isolated fungal endophytes would affect their host differently and their interaction would be modulated by AMF.

\section{Materials and methods}

\section{V. lychnitis seed germination}

Seeds used in the experiment were collected in autumn 2010 from $V$. lychnitis plants growing on the post-mining waste 
dump of the Trzebionka Mining Company (ZG Trzebionka) in close proximity to Chrzanów, PL (50 $09^{\prime} 19^{\prime \prime} \mathrm{N}, 19^{\circ} 25^{\prime} 10^{\prime \prime}$ E). Seeds were husked from capsules under a binocular microscope and surface sterilized with $8 \% \mathrm{NaOCl}$ (5 min), $96 \%$ ( $1 \mathrm{~min}$ ) and $75 \% \mathrm{EtOH}$ ( $3 \mathrm{~min}$; Achary et al. 2012, modified). Subsequently, seeds were rinsed for $1 \mathrm{~min} 5$ times in deionized water, dried on sterile filter paper, and germinated on water agar in Petri plates at room temperature, $12 \mathrm{~h}$ photoperiod and light intensity ca. $120 \mu \mathrm{mol} \times \mathrm{m}^{-2} \times \mathrm{s}^{-1}$ of PAR (photosynthetically active radiation).

\section{Inoculation with endophytes}

Ten-day-old seedlings were transferred to Petri plates (10 per each plate) filled with modified Strullu-Romand (MSR) medium (the concentration of GelGro ${ }^{\mathrm{TM}}$ (MP Biomedicals, FR) was increased to $11.0 \mathrm{~g} \times \mathrm{L}^{-1}$ instead of $3.0 \mathrm{~g} / \mathrm{L})$ without sucrose. Small plugs $(2 \times 2 \mathrm{~mm})$ of fresh endophyte mycelium were placed under the roots of seedlings. Plants were inoculated with one of the following endophytes: Pyrenophora leucospermi Crous and L. Swart, Cadophora sp., Diaporthe sp., Cochliobolus sativus (E.J. Butler) Shoemaker, Myrothecium sp., Xylaria sp., Phialophora mustea Neerg., and Phoma exigua var. exigua Desm. All fungi were isolated from shoots or roots of $V$. lychnitis plants (Wężowicz et al. 2014) and had been stored at $4{ }^{\circ} \mathrm{C}$ in the Małopolska Center of Biotechnolgy. Seedlings were grown with the endophytes for 28 days in a growth chamber (Percival AR-41L2X) at $21{ }^{\circ} \mathrm{C}, 12 \mathrm{~h}$ photoperiod, $60 \%$ humidity, and light intensity ca. $120 \mu \mathrm{mol} \times \mathrm{m}^{-2} \times \mathrm{s}^{-1}$ of PAR.

\section{Substrate preparation}

The substrate was collected from a deep layer (1 $\mathrm{m}$ depth) of the mine tailings, from sites devoid of plant cover for several years. The substratum was stored for 2 years before the experiment. The substratum contained practically no organic matter, was $\mathrm{P}$ - and $\mathrm{N}$-deficient $\left(P_{\text {total }}-900 \mu \mathrm{g} \times \mathrm{g}^{-1}, P_{\text {available }}\right.$ $\left.114 \mu \mathrm{g} \times \mathrm{g}^{-1} \mathrm{~N}-900 \mu \mathrm{g} \times \mathrm{g}^{-1}\right)$ and contained elevated quantities of toxic metals. The total $\mathrm{Zn}$ concentration reached $12,000 \mu \mathrm{g} \times \mathrm{g}^{-1}$ (ca. $3.8 \mu \mathrm{g} \mathrm{g}^{-1}$ after extraction with $\mathrm{Ca}\left(\mathrm{NO}_{3}\right)_{2}, \mathrm{~Pb}-2400 \mu \mathrm{g} \times \mathrm{g}^{-1}\left(1.9 \mu \mathrm{g} \times \mathrm{g}^{-1}\right.$ after extraction with $\left.\mathrm{Ca}\left(\mathrm{NO}_{3}\right)_{2}\right)$, and $\mathrm{Cd}-100 \mu \mathrm{g} \times \mathrm{g}^{-1}\left(1.4 \mu \mathrm{g} \times \mathrm{g}^{-1}\right.$ after extraction with $\mathrm{Ca}\left(\mathrm{NO}_{3}\right)_{2}$ (Orłowska et al. 2005). The $\mathrm{pH}$ was slightly alkaline ( $\mathrm{pH} 7.4)$. The substrate was not sterilized in order to replicate conditions in the tailings and also to investigate the possibility of use of the inoculum in recultivating the mine dump. The growth medium comprised the substrate, sterile sand, and expanded clay in a 3:1:1 $(v / v / v)$ ratio and was supplemented with rock phosphate $\left(1 \mathrm{~g} \times \mathrm{L}^{-1}\right)$.

\section{AM fungal inoculum preparation}

Rhizopagus irregularis (UNIJAG.PL.32 sub-cultured from BIORIZE E-1-99/Lav) inoculum, containing ca. 20 spores per gram of growth medium, was produced in pot cultures according to Jurkiewicz et al. (2010).

\section{Experimental design}

Seedlings were transferred to pots $\left(182 \mathrm{~cm}^{3}\right)$ filled with prepared growth medium (as described above). Where expected, AM fungal inoculum ( $2 \mathrm{~g}$ per pot) was added prior to seedlings transfer. The following treatments were prepared: (1) control seedlings - without mycorrhizal inoculum or endophytes (25 pots; NM controls); (2) seedlings inoculated with a single endophyte species (25 pots per each endophyte); (3) seedlings inoculated with an endophyte and $R$. irregularis (25 pots per each endophyte); and (4) seedlings inoculated with R. irregularis alone (25 pots). In each pot, a single plant was placed. Pots were irrigated once with $15 \mathrm{ml} \mathrm{KNO}_{3}$ solution (1.212 $\mathrm{g} / \mathrm{L})$ and cultivated in sealed sunbags (Sigma-Aldrich) in a greenhouse at $20{ }^{\circ} \mathrm{C}$, illuminated for $12 \mathrm{~h}$ with $120 \mu \mathrm{mol} \times \mathrm{m}^{-2} \times \mathrm{s}^{-1}$ of PAR. The pots were placed in trays, and the trays were rotated twice a week (each tray was rotated clockwise). The cultures were irrigated once per 2 weeks with $15 \mathrm{ml}$ of Long Ashton solution (Seddas-Dozolme et al. 2010) without phosphorus.

\section{Biometric measurements}

After 24 weeks of cultivation, plants were removed from pots and weighted. Roots were carefully washed in tap water before weighing. Survivability was calculated as frequency of plants (out of 25) that survived at the end of the experiment. Survivability data was arranged in a contingency table, and the chi-square test was used to evaluate statistical significance versus the NM controls. Roots were stained to visualize and assess mycorrhizal and endophyte colonization. Survivability and biomass production results were used to select plant-fungi consortia for analysis of photosynthesis efficiency, pigment/ protein abundance, and the impact of the presence of endophytic fungi on the development of mycorrhiza.

\section{Measurement of chlorophyll $a$ fluorescence transient OJIP}

Prior to plant harvesting, chlorophyll $a$ fluorescence transients OJIP were measured with a Handy PEA-fluorimeter (Hansatech Instruments, UK). Chlorophyll fluorescence is widely used to assess plant vitality (Strasser et al. 2004). The transients were induced by red light (peak at $650 \mathrm{~nm}$ ) of $3.000 \mu \mathrm{mol}$ photons $\mathrm{m}^{-2} \times \mathrm{s}^{-1}$ provided by an array of three light-emitting diodes and recorded for $1 \mathrm{~s}$ with 12 bit 
resolution. Data acquisition was performed every $10 \mu$ s (in the interval from $10 \mu \mathrm{s}$ to $0.3 \mathrm{~ms})$, every $0.1 \mathrm{~ms}(0.3-3 \mathrm{~ms})$, every $1 \mathrm{~ms}(3-30 \mathrm{~ms})$, every $10 \mathrm{~ms}(30-300 \mathrm{~ms})$, and every $100 \mathrm{~ms}$ (300 $\mathrm{ms}$ to $1 \mathrm{~s}$ ). The measurements were conducted on intact plants (8-15 replicates per treatment), dark-adapted for a minimum of $25 \mathrm{~min}$ prior to measurement.

For each treatment, the average OJIP fluorescence transient was analyzed according to the JIP test (Strasser et al. 2004), with the BIOLYZER5PHP software (http://www. fluoromatics.com). The parameters chosen to be calculated in the present study were the performance index $\left(\mathrm{PI}_{\mathrm{ABS}}\right)$, specific energy fluxes calculated per reaction center (TRo/ $\mathrm{RC}, \mathrm{ETo} / \mathrm{RC}, \mathrm{ABS} / \mathrm{RC}, \mathrm{RC} / \mathrm{AB}, \mathrm{DIo} / \mathrm{RC}$ ), area, quantum efficiencies, and flux ratios $\left(\phi_{\mathrm{Eo}}, \phi_{\mathrm{Po}}, \Psi \mathrm{o}\right)$. For parameter descriptions, see Table 1.

\section{AM fungi and endophyte colonization assessment}

Six plants and 15 fragments of each root system per treatment were evaluated for fungi colonization. The roots were stained according to the modified Phillips and Hayman (1970) method. After being washed in running tap water, roots were incubated in $10 \% \mathrm{KOH}$ solution for $24 \mathrm{~h}$ and rinsed in water, acidified in 5\% lactic acid solution for $1 \mathrm{~h}$, and stained with $0.05 \%$ aniline blue in $80 \%$ lactic acid for $24 \mathrm{~h}$ at room temperature. Finally, the material was transferred to $80 \%$ lactic acid for storage. For colonization assessment, roots were cut into $1.5 \mathrm{~cm}$ fragments and mounted on slides in glycerol/lactic acid (5:1). Analyses were carried out using a Nikon Eclipse 800 (Japan) microscope. Mycorrhizal colonization assessment was carried out according to Trouvelot's method (Trouvelot et al. 1986, http://www.dijon.inra.fr/bbceipm/Mychintec/ Mycocalc-prg/download.html). Observations of endophyte presence in roots were carried out simultaneously with mycorrhizal colonization assessment. Where necessary, to visualize the endophyte, additional staining with Sudan IV

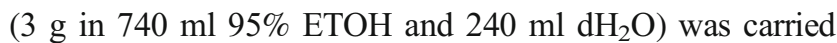
out on roots previously stained as above.

\section{SDS-PAGE and immuno-blotting}

To verify if fungi induced photosynthesis efficiency changes dependent on reorganization of photosyntem II antennae protein composition, the abundance of Lhcb3 which is one of three major chlorophyll $\mathrm{a} / \mathrm{b}$ binding proteins in photosystem II and the antennae Psbc protein was assessed. Additionally, the abundance of Rubisco LSU (large subunit), which catalizes the incorporation of $\mathrm{CO}_{2}$ into the Calvin-Asada cycle, was measured. Sample preparation was performed according to Rozpaciek et al. (2015) with modifications. Protein separation was performed in a $4 \%$ stacking gel $(\mathrm{pH}$ 6.8) and $12 \%$ resolving gel (pH 8.8) with $10 \mu \mathrm{g}$ of PsbC or Rubisco LSU or $20 \mu \mathrm{g}$ of Lhcb3 protein loaded per lane. Electrophoresis was carried out at constant $24 \mathrm{~mA}$ for the first $15 \mathrm{~min}$ (in the stacking gel), followed by $36 \mathrm{~mA}$ until full separation of the protein marker ladder (Thermoscientific, LT). Following electrophoresis, transfer to PVDF membranes (semi-dry) was performed using Trans-Blot Turbo Transfer System (BioRad, US). For immunodetection, Agrisera (SE) primary polyclonal antibodies in the following dilutions were used: PsbC (AS11 1787) 1:2500, Rubisco LSU (AS03 037) 1:5000, and Lhcb3 (AS01 002) 1:2000. After an overnight

Table 1 Terms used in the analysis of the OJIP fluorescence transient

\begin{tabular}{|c|c|}
\hline Symbol & Description \\
\hline $\mathrm{PI}_{\mathrm{ABS}}$ & $\begin{array}{l}\text { The performance of the photosynthesis apparatus expressed in relation to absorption. } \\
\text { Accounts for the density of reaction centers, the quantum yield of primary photochemistry, } \\
\text { and the ability to transfer electrons from PSII to PSI }\end{array}$ \\
\hline $\mathrm{TR}_{\mathrm{o}} / \mathrm{RC}$ & Energy capture by one reactive center \\
\hline $\mathrm{ET}_{\mathrm{o}} / \mathrm{RC}$ & Rate of the energy transfer by one reactive center \\
\hline $\mathrm{ABS} / \mathrm{RC}$ & The effective antenna size expressed as absorbance per reaction center \\
\hline $\mathrm{DI}_{\mathrm{o}} / \mathrm{RC}$ & The amount of energy dissipated per active reaction center \\
\hline Area & It is proportional to the concentration of electron acceptor pool size of PSII that includes QA, QB, and PQ \\
\hline QA & Primary quinone-type electron acceptors of PSII \\
\hline QB & Secondary quinone-type electron acceptors of PSII \\
\hline PQ & Plastoquinone-a quinone electron transporter \\
\hline$\phi_{\mathrm{Eo}}(\mathrm{ETo} / \mathrm{ABS})$ & The probability that an absorbed photon will move an electron into the electron transport chain \\
\hline$\phi_{\mathrm{Po}}(\mathrm{TRo} / \mathrm{ABS})$ & The maximal yield of primary photochemistry. When calculated from extreme values (Fo and Fm) Fv/Fm \\
\hline Fo & Fluorescence emitted when all reaction centers are open \\
\hline Fm & Fluorescence emitted when all reaction centers are closed \\
\hline $\mathrm{Fv} / \mathrm{Fm}$ & Current photochemical capacity of PSII \\
\hline$\Psi_{0}(\mathrm{ETo} / \mathrm{TRo})$ & Quantum yield of electron transport \\
\hline
\end{tabular}


incubation period at $4{ }^{\circ} \mathrm{C}$, membranes were washed with TBST and treated with secondary antibodies conjucted with alkaline phosphatase (Agrisera, SE) for $1.5 \mathrm{~h}$ (dilution 1:2000). Specific proteins were visualized by soaking membranes in 20\% BCIP/NBT (Sigma-Aldrich, USA) solution for $1 \mathrm{~min}$. After drying, membranes were scanned with an office scanner. Analyses were performed in triplicate. Densitometric analyses were performed with Image J (NIH, US).

\section{Chlorophyll concentration determination}

Chlorophyll concentration was measured according to Barnes et al. (1992).Chlorophyll $a$ and $b$ absorbance was measured at 665 and $648 \mathrm{~nm}$ with a spectrophotometer (BioTek, Q 5000, US).

\section{Statistical analyses}

Statistical significance was determined with Student's $t$ test $(p \leq 0.05)$, with the exception of $V$. lychnitis survivability results, for which data were arranged in a contingency table and the chi-square test was used. Additionally, a two-way ANOVA was performed to evaluate the effect of double inoculation on plant biomass. Data normality and variance homogeneity were evaluated by the Shapiro-Wilk and Levine's tests, respectively. Statistical analyses were conducted using STATISTICA ver. 10 (Statsoft).

\section{Results}

\section{Biometric measurements}

Inoculation of V. lychnitis with Cadophora sp., Diaporthe sp., Myrothecium sp., P. leucospermii, and P. mustea resulted in a significant decrease in biomass production. Xylaria sp., C. sativus, and P. exigua var. exigua did not affect V. lychnitis growth (Fig. 1a). The addition of mycorrhizal fungi to the setup significantly affected all of the plant-endophyte consortia: M plants yielded more biomass compared to their solely endophyte-inoculated counterparts. The effect of mycorrhiza also was beneficial for control non-inoculated plants. Dual inoculation with AMF and C. sativus, Diaporthe sp., and P. exigua var. exigua gave the greatest biomass yield (statistically significant increase compared to the $\mathrm{M}$ control), whereas inoculation with the remaining endophytes did not significantly affect the growth of $V$. lychnitis compared to the $\mathrm{M}$ control.

Survivability of NM plants inoculated with Diaporthe sp., Myrothecium sp., and P. leucospermii was significantly decreased. Cadophora sp., C. sativus, P. exigua var. exigua,

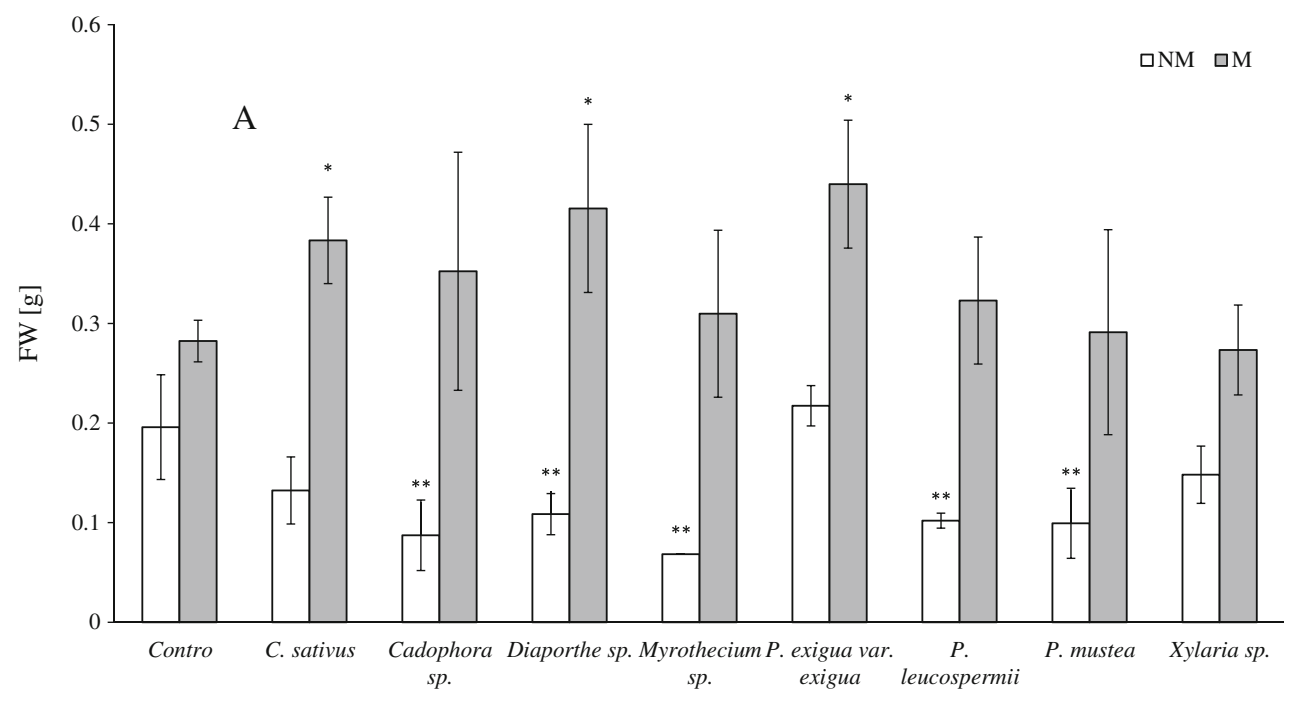

Fig. 1 Fresh weight (FW) of V. lychnitis inoculated with a single endophyte (white bars; NM) and subject to inoculation with AMF and endophytic fungi (gray bars; M). Single stars indicate statistically significant differences between $V$. lychnitis inoculated with both types of fungi and the M control. Double stars indicate statistically significant differences between the NM control and plants inoculated with a single

\section{B}

Endophyte*Mycorrhiza $\mathrm{p}=0,00715$

Endophyte $\mathrm{p}=0,00101$

Mycorrhiza $\mathrm{p}=0,00000$

fungal endophyte. Biomass production was lower in NM than M plants in all of the setups tested. Statistical significance was assessed by the $t$ test, $P \leq 0.05$. Whiskers represent SD from $N=3-22$ (a). Two-way ANOVA was performed to evaluate the effect of double inoculation on plant biomass $(\mathbf{b})$ 
Xylaria sp., and P. mustea did not affect the survival of NM plants. Co-inoculation with AMF significantly improved the survival rate of $V$. lychnitis. In all of the plant-fungi constoria tested except $P$. exigua var. exigua, the number of dualinoculated plants that survived the experiment was significantly higher compared to the NM control (Fig. 2).

\section{Selection of plant-fungi consortia for further analysis}

For more detailed analysis of the interaction between the plant with fungal endophytes and the plant-endophyte-AMF association, plant-microbe consortia were selected according to the biomass production and survivability results. Xylaria sp. was selected because it was the only species that did not affect the plants' biomass production in both single and coinoculation experiments, nor did it alone influence the survivability of $V$. lychnitis. Single inoculation with $P$. exigua var. exigua neither affected plant growth nor survivability, but coinoculation with AMF resulted in the highest plant biomass yield among the endophytic fungi tested. It also was the only species that did not improve plant survivability in coinoculation experiments. The responses of $V$. lychnitis to Diaporthe sp. were the most diverse among the tested endophytes, depending on the presence of AMF. In single inoculation experiments, Diaporthe sp. clearly hindered plant growth and survivability, but co-inoculation with AMF significantly improved all biometric parameters tested. The selection of Xylaria sp., P. exigua var. exigua and Diaporthe sp. allowed us to examine the response of the photosynthesis apparatus to fungi that affect plant productivity differently, depending on co-inoculation. We anticipated that alterations of growth would reflect photosynthesis efficiency and pigment/structural protein composition of photosystem II.

\section{AM fungi and endophyte colonization}

Among V. lychnitis: (1) non-inoculated; (2) inoculated with endophytes Diaporthe sp., Xylaria sp., or P. exigua var. exigua; (3) inoculated with $R$. irregularis and endophytes Diaporthe sp., Xylaria sp,. or P. exigua var. exigua; and (4) inoculated only with $R$. irregularis, arbuscular mycorrhizas with arbuscules and abundant vesicles were observed in all mycorrhiza-inoculated plants. In plants inoculated with fungal endophytes, thin hyphae of Diaporthe sp., Xylaria sp., and P. exigua var. exigua were observed in roots (Fig. S1) growing narrowly between cortical cells. Their dyeability, however, was lower than that of AMF hyphae from the same area of the root. Mycorrhizal colonization parameters are presented in Fig. 3. Mycorrhizal colonization (M\%) of V. lychnitis inoculated with Xylaria sp. exhibited a significant increase (7\%) in comparison to AMF control plants. M plants inoculated with Diaporthe sp. showed a significant decline (13\%) in A\%. In root samples of NM V. lychnitis, no mycorrhizal structures and endophytes were found.

\section{Photosynthesis efficiency}

Among the endophytes selected for further analysis, single inoculation with Diaporthe sp. resulted in significant declines (compared to the $\mathrm{NM}$ control) in $\mathrm{PI}_{\mathrm{ABS}}(54 \%), \phi \mathrm{Po}(7 \%)$, and $\phi$ Eo (11\%) and an upregulation of TRo/RC (12\%), ABS/RC (20\%), and Dio/RC (51\%). Xylaria sp. inoculated plants

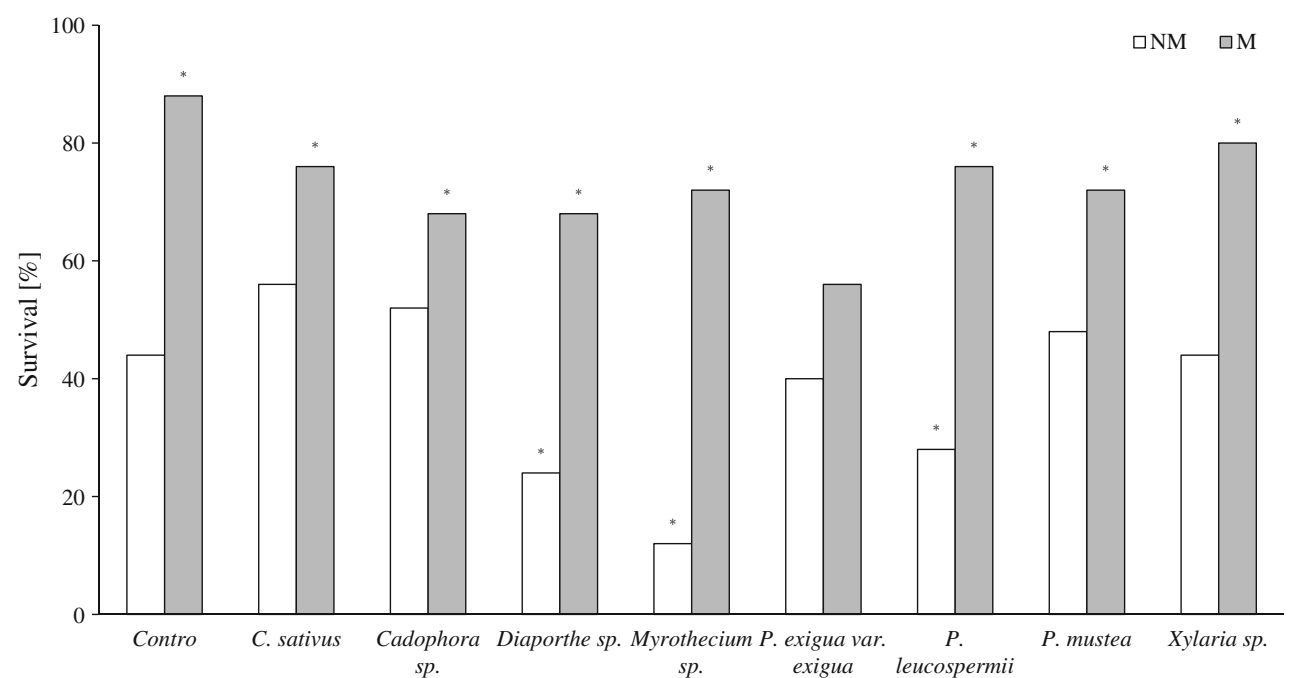

Fig. 2 The survival percentage of $V$. lychnitis inoculated with an endophytic fungus (white bars; NM) and co-inoculated with AMF and an endophytic fungus (gray bars; M) through 24 weeks of growth in substratum from the mine dump "Bolesław." Stars indicate statistically significant differences between inoculated $V$. lychnitis and the NM control. The results were arranged in a contingency table and the chi-square test was used to evaluate statistical significance, $P \leq 0.05$ 


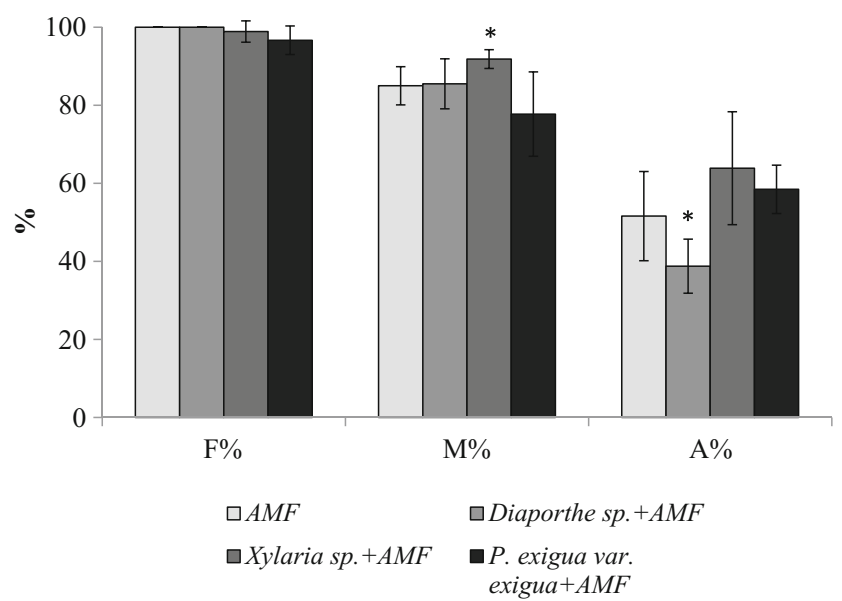

Fig. 3 Effect of endophytes (Diaporthe sp., Xylaria sp. and P. exigua var. exigua) on arbuscular mycorrhizal colonization parameters of $V$. lychnitis inoculated with $R$. irregularis (AMF). The depicted parameters arethe following: $F(\%)$ mycorrhizal colonization frequency, $M(\%)$ relative mycorrhizal colonization intensity, and $A(\%)$ arbuscule abundance. Plants were cultivated in a greenhouse in substratum composed of a mixture of tailings material, sand, and expanded clay at 3:1:1(v/v/v). The numbers are given in percent according to the parameter definitions by Trouvelot et al. (1986). Bars represent mean values from 6 independent measurements, $N=6$. Asterisks above the columns indicate statistically significant differences ( $t$ test) between each treatment and $\mathrm{M}$ control $(P \leq 0.05)$

showed a significant reduction of the area parameter (19\%) and a significant increase of $\Psi 0(8 \%), \phi E o(9 \%)$, and a $21 \%$ in $\mathrm{PI}_{\mathrm{ABS}}$ in comparison with $\mathrm{NM}$ control plants. Photosynthesis efficiency was not affected significantly by inoculation with P. exigua var. exigua (Fig. 4a).

In M plants, inoculation with Diaporthe sp. induced a significant increase (compared to NM control plants) of $\phi E$ Eo and $\mathrm{e} \Psi_{\mathrm{o}}$ (both 7\%) which translated into an upregulation of $\mathrm{PI}_{\mathrm{ABS}}$. Co-inoculation with Xylaria sp. or P. exiqua var. exigua and AMF did not affect photosynthesis efficiency (Fig. 4b).

\section{Rubisco and photosystem II protein abundance}

Among plants inoculated with endophytes, the abundance of PsbC was decreased by $30 \%$ in Diaporthe sp-colonized plants versus NM controls. Xylaria sp. and P. exigua var. exigua did not significantly affect the abundance of PsbC. Co-inoculation with AMF significantly increased $\mathrm{PsbC}$ abundance in all plants tested. Diaporthe sp., Xylaria sp., and P. exigua var. exigua increased PsbC abundance by 40,43 , and $52 \%$, respectively, compared to NM control plants. No significant differences in Rubisco and in Lhcb3 abundance were found in colonized plants, compared to NM control plants (Fig. 5a).

\section{Chlorophyll concentration}

In NM plants inoculated with Diaporthe sp. and Xylaria sp., a significant increase (2.5-fold and 2-fold, respectively) in Chl b
A

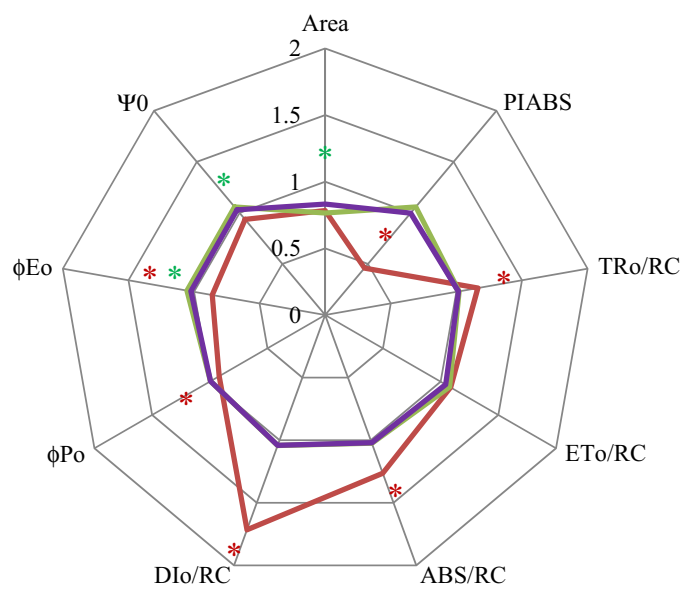

B

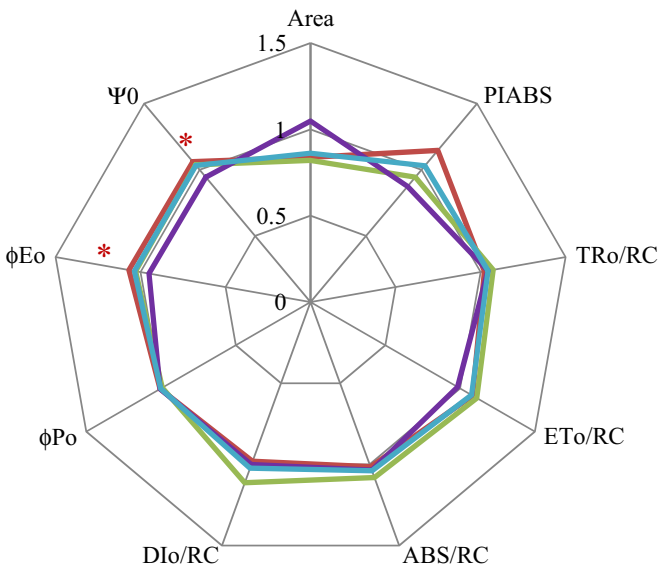

Diaporthe $s p . \quad$ Xylaria $s p . \quad \longrightarrow$. exigua var. exigua $\quad$ -

Fig. 4 "Spider plots"of selected fluorescence parameters describing energy fluxes in PSII in a plants inoculated with endophytes and $\mathbf{b}$ plants inoculated with endophytes and $R$. irregularis (AMF) The values were normalized to those of the NM control plant (i.e., NM controls have a value of 1 for each parameter). Definitions of the parameters in Table 1 Asterisks in colors corresponding to the line for each fungus indicate statistically significant ( $t$ test) differences between each treatment and the NM control $(P \leq 0.05)$. Whiskers represent SD from $N=5$

concentration, compared to NM control plants, was observed (Fig. 5b). P. exigua var. exigua did not affect $\mathrm{Chl} b$ concentration (Fig. 5b). No differences in total chl and Chla were observed in NM plants. In M plants inoculated with Xylaria sp., a significant increase in total Chl (almost 2-fold increase), in Chl a (0.5-fold), and in Chl b (almost 4-fold), compared to NM control plants, was found. In the remaining treatments, no significant differences were observed (Fig. 5b).

\section{Discussion}

The role of fungal endophytes in plant physiology and their possible utilization as growth promotion and stress protection agents recently has been a popular subject of research. Here, we report that, under laboratory conditions, fungal endophytes 
Fig. 5 a Relative PsbC, Rubisco, Lhcb3 content from $V$. lychnitis. Bars represent mean values from three independent measurements, $N=3$. The values were normalized relative to those of the NM control plant. b Total concentrations from $V$. lychnitis. Bars represent mean values from seven independent inoculated with endophytes and/ or inoculated with $R$. irregularis (AMF) Asterisks above the bars indicate statistically significant differences ( $t$ test) between each $(P \leq 0.05)$. Whiskers represent SD chlorophyll, chlorophyll a and b measurements, $N=7$. Plants were treatment and the NM control
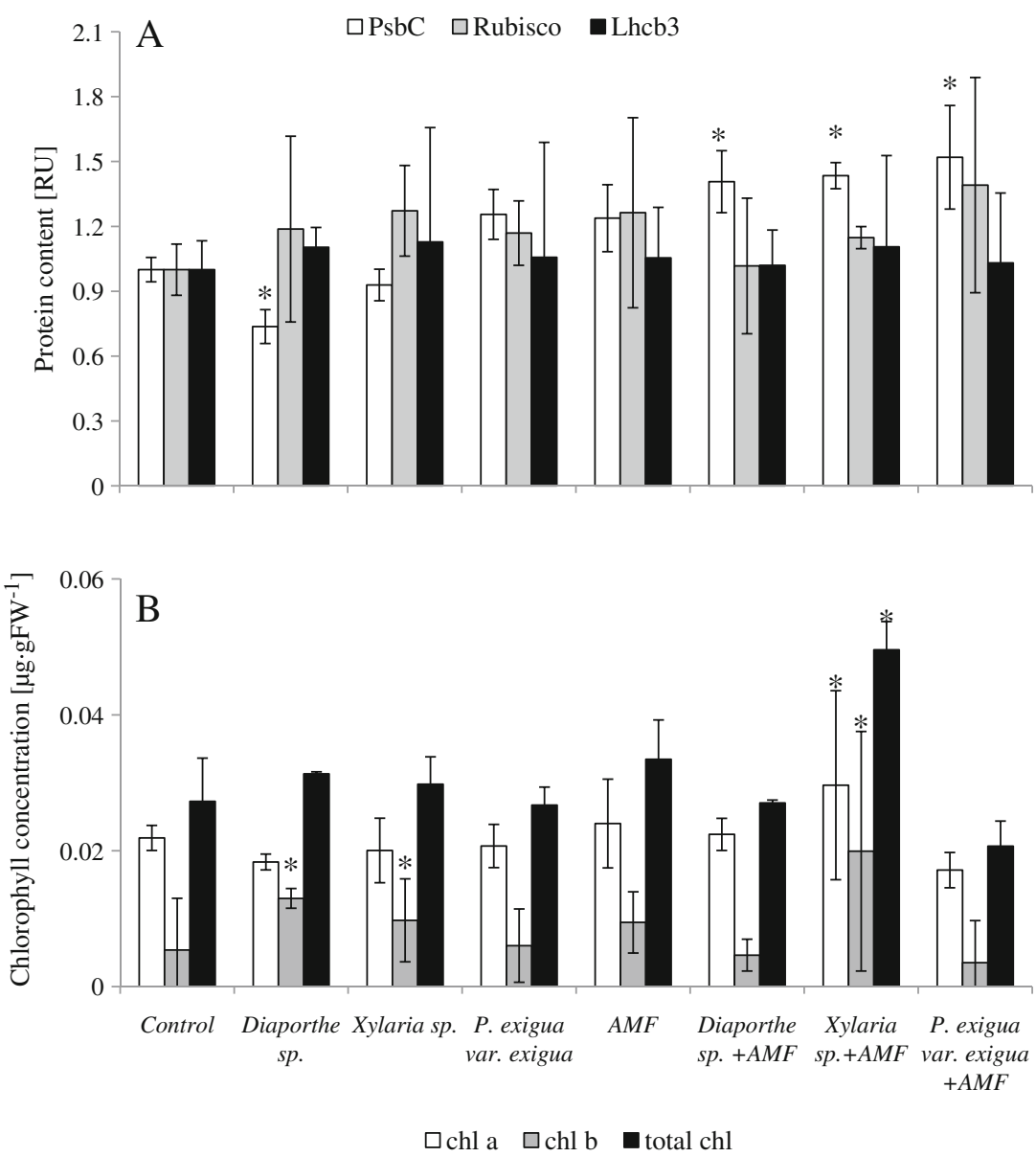

did not promote $V$. lychnitis growth in substrate from a postmining waste dump. In single inoculation experiments, plant fitness, in terms of biomass production and leaf area (data not shown), was negatively affected by 5 out of the 8 endophytes tested. The remaining three fungi had no effect on plant growth. It seems that reduced growth was the cost of maintaining the endophytes within plant tissues. AMF in this setup remarkably improved the growth parameters of the endophyte-inoculated plants. In all plant-fungi consortia examined, biomass production and survivability (excluding P. exigua var. exigua) was improved compared to the NM control, suggesting that AMF positively modulates the plantfungal endophyte interaction. $V$. lychnitis yielded the highest biomass when co-inoculated with AMF and C. sativus, Diaporthe sp., and P. exigua var. exigua. The survival rate of $M$ plants co-inoculated with endophytes was notably higher than the NM control, but not as high as the AMF control (with no endophyte colonization). This probably can be attributed to the methodology used in the experiment. Inoculation with the endophyte took place under in vitro conditions, 4 weeks before inoculation with AMF to ensure endophyte colonization. It seems as though that the attenuating effect of AMF was not sufficient enough to allow all the plants inoculated with endophytes 4 weeks earlier to survive. In order to gain a comprehensive understanding of the endophyte-induced growth dynamics, a different experimental setup (simultaneous inoculation with AMF and fungal endophytes) is necessary. $V$. lychnitis is a biennial herb, which grows up to $2 \mathrm{~m}$ in height in its second year. To describe the role of symbiotic fungi in V. lychnitis fitness, more thorough analysis of plant growth, seed production, etc. after two growth seasons is required. Another issue that needs to be raised is that $V$. lychnitis in natural conditions is almost exclusively found associated with mycorrhiza (unpublished data). This suggests that any depletion of the mycorrhizal partner may have negatively affected the plant's physiology, which, in turn, may have affected its interaction with the endophyte. Overall, V. lychnitis could only benefit from the endophytic fungi in its mycorrhizal state.

The mechanisms by which mycorrhizas improve plant growth and fitness under metal toxicity have been reviewed recently (Ruytinx et al. 2016). The majority of data describing the beneficial role of AMF under metal toxicity comes from experiments conducted under controlled conditions, or that did not recognize the potential role of other symbiotic organisms in mycorrhiza-dependent toxic metal stress alleviation, even though in natural environments plants are inhabited by a 
multitude of microorganisms. In this study, we examined the growth response of $V$. lychnitis in both single inoculation experiments and in co-inoculation experiments with both AMF and fungal endophytes. We found that co-inoculation with certain species may facilitate plant growth in a toxic metalenriched environment. The models used in this study do not exhaust all the possible interactions of $V$. lychnitis in nature, but are a step forward in understanding the role of different microorganisms in plant biology. To elucidate the mechanisms of this tripartite interaction, further research is necessary.

According to the available literature, the fungal endophytes tested in this study usually establish beneficial or neutral associations with their hosts. Being one of the most dominant endophytes, Diaporthe (Phomopsis) species are commonly isolated as endophytes from different dicot plant hosts (Suryanarayanan et al. 2002; Sun et al. 2011). Some species of Diaporthe, however, show either pathogenic or mutualistic interactions with plants depending on the host and its health; for example, $D$. phaseolorum is pathogenic to soybean (Santos et al. 2011), but endophytic in mangroves (Sebastianes et al. 2011). Diaporthe spp. have been reported to cause a wide range of plant diseases (Scheper et al. 2000; Heller and Gierth 2001). Xylaria species occur on a broad diversity of plant hosts; however, they are frequently isolated from wood tissues (Frohlich et al. 2000; Wang et al. 2014). Fungi from this genus present fungal activity against plant pathogens (Park et al. 2005; Fukasawa et al. 2009) but also show strong capacity to degrade cellulose and lignin (Osono and Takeda 2002). This strategy may be adapted for a saprotrophic lifestyle. Species from the Phoma genus are asymptomatically present in numerous plants: Junker et al. (2012) isolated this fungus along with other fungi from wild Arabidopsis thaliana in which it did not cause any disease symptoms. In a single inoculation in vitro experiment, however, Phoma sp. parasitized Arabidopsis, suggesting that the A. thaliana-Phoma sp. interaction is modulated by the plant microbiota (in vitro conditions may had also affected the interaction). A similar relationship was reported by Larimer et al. (2010) who showed that fungal foliar endophytes decreased plant performance; however, co-inoculation with AMF attenuated the negative effect of endophytes.

The growth promoting role of fungal endophytes, under limited resource availability, is controversial. Studies performed on Festuca grass species and the Neothyphodium/ Epichloe endophytes give conflicting results (Ahlholm et al. 2002). In Ahlholm et al.'s (2002) experiment, fungus species previously described as neutral and mutualistic endophytes severely affected the growth of their host. Endophytic fungi acquire all necessary growth and maintenance resources from their host plant, thereby affecting resource transport from source to sinks and allocation within the plant (Ahlholm et al. 2002). Vegetation in conditions of limited resource availability, such as a post-mining waste dump, severely hinder plant performance. An additional sink probably exceeded the host ability to maintain its own vegetative functions. The potential benefits coming from the presence of the endophyte did not compensate the costs of the symbiosis leading to decreased plant biomass production. In contrast to fungal endophytes, AMF hyphae extend beyond the rhizosphere. This allows AMF to aquire resources independent of plant growth. Mineral nutrients acquired by the fungi may be translocated to the plant. We speculate that the endophyte confined to plant tissues was not efficient in provisioning its host, which resulted in growth retardation. The presence of the AMF in the setup remarkably improved plant growth, suggesting that the benefits (nutrient acquisition, stress protection) provided by the AMF were sufficient to allow the plant to provide nutrients to all its symbiotic partners. Additionally, a role in modulating endophytic fungi growth and lifestyle by AMF cannot be excluded, but this requires further research.

Toxic metals significantly affect the performance of the photosynthetic apparatus, limiting the efficiency of carbon assimilation, thus affecting the overall nutritional status of the plant. Limiting the production of the "source" forces the "sinks" to adapt. An additional energy sink, the endophyte, requires the plant to share its carbon resources with its symbiotic partner. Under optimal conditions, plant production may exceed its needs, allowing it to allocate a share of its photoassimilates to the fungi. In photosynthesis limiting conditions, however, the plant suffers from carbon deficiencies (with a consequent slower growth rate, diminished ability to counteract stress-induced damage, etc.) hindering its ability to invest in additional sinks for resources. Aloui et al. (2011) showed that Glomus irregulare alleviated the negative effects of Cd on photosynthesis in Medicago truncatula by increasing the plant's ability to utilize light energy and by facilitating electron transport. According to Rozpądek et al. (2015), colonization of orchard grass by Epichloe typhina led to changes in the host's photosynthetic apparatus, e.g., increased electron density flux, increased stomatal conductance, and an abundance of $\mathrm{Chl} b$ and PSI and PSII proteins. As a result, PSII photochemistry efficiency, carbon assimilation, and light harvesting capacity in plants were improved, allowing orchard grass to cope with endophyte energy demands while also sustaining orchard grass growth.

In single inoculation experiments with Diaporthe sp., V. lychnitis exhibited a significant decline in photosynthesis performance. The efficiency of energy production in relation to absorption, manifested as electron flow in PSII ( $\mathrm{PI}_{\mathrm{ABS}}$ ), decreased by close to $50 \%$. The photosynthesis index $\mathrm{PI}_{\mathrm{ABS}}$ combines three variables indicative of different processes associated with PSII photochemistry: the density of reaction centers, the quantum yield of primary photochemistry, and the ability to transfer electrons from PSII to PSI (Corrêa et al. 2006). In single inoculation with Diaporthe sp., the photochemistry of PSII was decreased due to a significant 
decline in the efficiency of primary photochemistry $(\varphi \mathrm{E}$, $\varphi \mathrm{Po})$. Even though, the light-harvesting capacity was improved $(\mathrm{ABS} / \mathrm{RC})$, the photosynthetic apparatus was unable to transform incident light into energy (lower electron transport efficiencies) necessary for carbon assimilation. These results suggest that even though solar energy was absorbed, it was not transformed into fixed energy, but instead was dissipated as heat (DIo/RC). Interestingly, inoculation with mycorrhiza (co-inoculation with AMF) restored to control levels, or even above control levels, all the photosynthesis indicies that were negatively affected by single inoculation with Diaporthe $\mathrm{sp}$. The restoration was correlated with a significant growth improvement. In the case of Xylaria sp.-inoculated plants (single inoculation) which did not respond to the endophyte growth-wise, a significant improvement in $\varphi$ Eo and $\Psi 0$ was found, but this did not translate into elevated overall photosynthesis efficiency $\left(\mathrm{PI}_{\mathrm{ABS}}\right)$. According to previous reports, inoculation with mycorrhiza improves plant growth in toxic metal-enriched habitats. Higher biomass yield often is correlated with improved photosynthesis performance (Rozpadek et al. 2014, reviewed in Ruytinx et al. 2016). In this study, however, mycorrhizas did not affect the photosynthesis efficiency of $V$. lychnitis, suggesting that accelerating the carbon assimilation rate was not necessary for improved growth. It also was not required for provisioning its symbiotic partners, indicating that the energy demands of the plant-microbe consortium were met by means other than improved photosynthesis efficiency.

The role of toxic metals in chlorophyll (Chl) biosynthesis and stability has been comprehensively described. Toxic metals were reported to inhibit chlorophyll biosynthesis (Ouzounidou 1995). $\mathrm{Pb}$ was reported to inhibit chlorophyll biosynthesis by causing impaired uptake of essential elements such as $\mathrm{Mg}$ and Fe by plants (Burzyński and Grabowski 1984). Chl $b$ is more vulnerable to $\mathrm{Pb}$ toxicity than $\mathrm{Chl} a$ (Vodnik et al. 1999). Pb changes the lipid composition of thylakoid membranes (Stefanov et al. 1995) and damages the photosynthetic apparatus due to its affinity for protein Nand S-ligands (Ahmed and Tajmir-Riahi 1993). Cd is known to decrease the total Chla/b ratio in plants (Sheoran et al. 1990) and to suppress chloroplast development (Malik et al. 1992a, b). Here, inoculation with endophytes Xylaria sp. and Diaporthe sp. significantly increased the abundance of $\mathrm{Chl} b$. Diaporthe sp. also increased energy absorption and flow through a single reaction center (although it did not translate into energy production). Chl $b$ plays an important role in the assembly of light-harvesting complexes and in the structure of reaction centers. In a previous study, fungal endophytes were found to alter the composition of photosynthetic pigments and light harvesting center proteins (LHC), including the relative abundance of $\mathrm{Chl} b$, $\mathrm{PsbC}$, and other proteins involved in light harvesting and processing. This was accompanied by improved photosynthesis efficiency (Rozpądek et al. 2015).
Higher energy fluxes potentially accelerate energy production in PSII. In this experiment, however, probably due to plant growth conditions, structural changes did not translate into enhanced performance. In order to map endophyte-induced changes in the composition of photosynthesis functional proteins, we tested those shown previously to be affected by Dactylis glomerata inhabited by E. typhina and others. Unfortunately, due to methodology issues (the antibodies available did not react with epitopes from $V$. lychnitis proteins or did not allow us to perform a semi-quantitative analysis), we were not able to quantify the abundance of other photosynthesis-related proteins. Those that we quantified did not exhibit significant changes upon inoculation. Our results indicated that only the abundance of the LHC structural PsbC protein increased in $\mathrm{M}$ plants co-inoculated with endophytes, but not in single inoculation experiments with endophytic fungi or AMF. Because inoculation with the AMF alone did not yield such results, we propose that increasing the abundance of PsbC is an endophyte-specific feature, but in the case of $V$. lychnitis, only plants co-inoculated with AMF (V. lychnitis is mycorrhizal in nature) exhibit such an alteration. The abundance of the remaining proteins (Rubisco LSU and Lhcb3) evaluated in this study did not change in any of the experimental setups. This suggest that even though the presence of the endophytes did affect the composition of photosynthetically active pigments and proteins in V. lychnitis, these changes were insufficient to improve photosynthesis performance.

\section{Conclusions}

Studies investigating interactions between fungal endophytes, AMF, and non-grass hosts have received little attention until recently. The experiments conducted here showed that $V$. lychnitis did not benefit from single inoculation with endophytes in a substrate containing toxic metals. Inoculation with both AMF and endophytes significantly improved plant growth parameters. Particularly, V. lychnitis co-inoculated with AMF and C. sativus, Diaporthe sp., and P. exigua var. exigua yielded the highest biomass, exceeding the growth rate of both non-inoculated and AMF plants. These results, hopefully, can be utilized in phytoremediation strategies. Inoculation with the fungi tested did not affect photosynthesis efficiency. Interestingly, however, in the beneficial setups that included fungal endophytes, the abundance of PsbC was increased. This indicates that upon colonization with endophytic fungi, changes in the composition of proteins involved in light harvesting do take place. Previously, this has been found to be associated with an improvement of photosynthesis efficiency. 
Acknowledgments This work was supported by The National Science Center (MAESTRO project) on the basis of DEC-2011/02/A/NZ9/00137 and grants from the Jagiellonian University (DS/MND/WBiNoZ/INoŚ/ 26/2012, DS/MND/WBiNoZ/INoŚ/34/2013).

\section{Compliance with ethical standards}

Conflict of interest The authors declare that they have no conflict of interest.

Open Access This article is distributed under the terms of the Creative Commons Attribution 4.0 International License (http:// creativecommons.org/licenses/by/4.0/), which permits unrestricted use, distribution, and reproduction in any medium, provided you give appropriate credit to the original author(s) and the source, provide a link to the Creative Commons license, and indicate if changes were made.

\section{References}

Achary VMM, Patnaik AR, Panda BB (2012) Oxidative biomarkers in leaf tissue of barley seedlings in response to aluminum stress. Ecotoxicol Environ Saf 75:16-26

Ahlholm JU, Helander M, Lehtimäki S, Wäli P, Saikkonen K (2002) Vertically transmitted fungal endophytes: different responses of host-parasite systems to environmental conditions. Oikos 99:173183

Ahmed A, Tajmir-Riahi HA (1993) Interaction of toxic metal ions $\mathrm{Cd}^{2+}$, $\mathrm{Hg}^{2+}$ and $\mathrm{Pb}$ with light-harvesting proteins of chloroplast thylakoid membranes. An FTIR spectroscopic study. J Inorg Biochem 50: 235-243

Aloui A, Recorbet G, Robert F, Schoefs B, Bertrand M, Henry C, Gianinazzi-Pearson V, Dumas-Gaudot E, Aschi-Smiti S (2011) Arbuscular mycorrhizal symbiosis elicits shoot proteome changes that are modified during cadmium stress alleviation in Medicago truncatula. BMC Plant Biol 11:75

Aly AH, Debbab A, Proksch P (2011) Fungal endophytes: unique plant inhabitants with great promises. Appl Microbiol Biotechnol 90: 1829-1845

Barnes JD, Balaguer L, Manrique E, Elvira S, Davison AW (1992) A reappraisal of the use of DMSO for the extraction and determination of chlorophylls $\mathrm{a}$ and $\mathrm{b}$ in lichens and higher plants. Environ Exper Bot 32:85-100

Beckett DC, Aartila TP, Miller AC (1992) Invertebrate abundance on Potamogeton nodosus: effects of plant surface area and condition. Can J Zoolog 70:300-306

Bever JD, Richardson SC, Lawrence BM, Holmes J, Watson M (2009) Preferential allocation to beneficial symbiont with spatial structure maintains mycorrhizal mutualism. Ecol Lett 12:13-21

Burzyński M, Grabowski A (1984) Influence of lead on nitrate uptake and reduction in cucumber seedlings. Acta Soc Bot Pol 53:77-86

Cordier C, Pozo MJ, Barea JM, Gianinazzi S, Gianinazzi-Pearson V (1998) Cell defense responses associated with localized and systemic resistance to Phytophthora parasitica induced in tomato by an arbuscular mycorrhizal fungus. Mol Plant-Microbe Interact 11: $1017-1028$

Corrêa A, Strasser RJ, Martins-Loução MA (2006) Are mycorrhiza always beneficial? Plant Soil 279:65-73

Daei G, Ardekani MR, Rejali F, Teimuri S, Miransari M (2009) Alleviation of salinity stress on wheat yield, yield components, and nutrient uptake using arbuscular mycorrhizal fungi under field conditions. J Plant Physiol 166:617-625

de Andrade Lopez SA, Domingues AP, Mazzafera P (2015) Photosynthesis is induced in rice plants that associate with arbuscular mycorrhizal fungi and are grown under arsenate and arsenite stress. Chemosphere 134:141-149

Deng Z, Cao L, Huang H, Jiang X, Wang W, Shi Y, Zhang R (2011) Characterization of $\mathrm{Cd}$ - and $\mathrm{Pb}$-resistant fungal endophyte $\mathrm{Mucor}$ sp. CBRF59 isolated from rapes (Brassica chinensis) in a metalcontaminated soil. J Hazard Mater 185:717-724

Doan TT, Jäschke D, Ludwig-Müller J (2008) An endophytic fungus induces tolerance against the clubroot pathogen Plasmodiophora brassicae in Arabidopsis thaliana and Brassica rapa roots. In: V International Symposium on Brassicas and XVI International Crucifer Genetics Workshop, Brassica 2008 867, pp 173-180

Felton GW, Tumlinson JH (2008) Plant-insect dialogs: complex interactions at the plant-insect interface. Curr Opin Plant Biol 11:457-463

Frohlich J, Hyde KD, Petrini O (2000) Endophytic fungi associated with palms. Mycol Res 104:1202-1212

Fukasawa Y, Osono T, Takeda H (2009) Effects of attack of saprobic fungi on twig litter decomposition by endophytic fungi. Ecol Res 24:1067-1073

Heller A, Gierth K (2001) Cytological observations of the infection process by Phomopsis helianthi (Munt.-Cvet) in leaves of sunflower. J Phytopathol 149:347-357

Hiruma K, Gerlach N, Sacrista S, Bucher M, O'Connell RJ, SchulzeLefert P (2016) Root endophyte Colletotrichum tofieldiae confers plant fitness benefits that are phosphate status dependent. Cell 165: 464-474

Jäschke D, Dugassa-Gobena D, Karlovsky P, Vidal S, Ludwig-Müller J (2010) Suppression of clubroot (Plasmodiophora brassicae) development in Arabidopsis thaliana by the endophytic fungus Acremonium alternatum. Plant Pathol 59:100-111

Junker C, Draeger S, Schulz B (2012) A fine line-endophytes or pathogens in Arabidopsis thaliana. Fungal Ecol 5:657-662

Jurkiewicz A, Ryszka P, Anielska T, Waligórski P, Białońska D, Góralska K, Tsimilli-Michael M, Turnau K (2010) Optimization of culture conditions of Arnica montana L.: effects of mycorrhizal fungi and competing plants. Mycorrhiza 20:293-306

Kiers ET, Palmer TM, Ives AR, Bruno JF, Bronstein JL (2010) Mutualisms in a changing world: an evolutionary perspective. Ecol Lett 13:1459-1474

Larimer AL, Bever JD, Clay K (2010) The interactive effects of plant microbial symbionts: a review and meta-analysis. Symbiosis 51: 139-148

Larimer AL, Bever JD, Clay K (2012) Consequences of simultaneous interactions of fungal endophytes and arbuscular mycorrhizal fungi with a shared host grass. Oikos 121:2090-2096

Lewis GC (2004) Effects of biotic and abiotic stress on the growth of three genotypes of Lolium perenne with and without infection by the fungal endophyte Neotyphodium lolii. Ann Appl Biol 144:53-63

Leyval C, Turnau K, Haselwandter K (1997) Effect of heavy metal pollution on mycorrhizal colonization and function: physiological, ecological and applied aspects. Mycorrhiza 7:139-153

Li HY, Li DW, He CM, Zhou ZP, Mei T, Xu HM (2011a) Diversity and heavy metal tolerance of endophytic fungi from six dominant plant species in a Pb-Zn mine wasteland in China. Fungal Ecol 5:309-315

Li T, Liu MJ, Zhang XT, Zhang HB, Sha T, Zhao ZW (2011b) Improved tolerance of maize (Zea mays L.) to heavy metals by colonization of a dark septate endophyte (DSE) Exophiala pisciphila. Sci Total Environ 409:1069-1074

Li HDY, Wei DQ, Shen M, Zhou ZP (2012) Endophytes and their role in phytoremediation. Fungal Divers 54:11-18

Lindow SE, Brandl MT (2003) Microbiology of the phyllosphere. Appl Environ Microb 69:1875-1883

Mack M, Rudgers JA (2008) Balancing multiple mutualists: asymmetric interactions among plants, arbuscular mycorrhizal fungi, and fungal endophytes. Oikos 117:310-320 
Malik O, Sheoran IS, Singh R (1992a) Lipid composition of thylakoid membranes of cadmium treated wheat seedlings. Indian J Biochem Biophys 29:350-354

Malik O, Sheoran IS, Singh R (1992b) Carbon metabolism in leaves of cadmium treated wheat seedlings. Plant Physiol Biochem 30:223229

Mandyam KG, Roe J, Jumpponen A (2013) Arabidopsis thaliana model system reveals a continuum of responses to root endophyte colonization. Fungal Biol 117:250-260

Orłowska E, Ryszka P, Jurkiewicz A, Turnau K (2005) Effectiveness of arbuscular mycorrhizal fungal (AMF) strains in colonisation of plants involved in phytostabilisation of zinc wastes. Geoderma 129:92-98

Osono T, Takeda H (2002) Comparison of litter decomposing ability among diverse fungi in a cool temperate deciduous forest in Japan. Mycologia 94:421-427

Ouzounidou G (1995) Cu-ions mediated changes in growth, chlorophyll and other ion contents in a cu-tolerant Koeleria splendens. Biol Plant 37:71-79

Park JH, Choi GJ, Lee HB, Kim KM, Jung HS, Lee SW, Jang KS, ChoKY KJC (2005) Griseofulvin from Xylaria sp. strain F0010, an endophytic fungus of Abies holophylla and its antifungal activity against plant pathogenic fungi. J Microbiol Biotechnol 15:112-117

Phillips JM, Hayman DS (1970) Improved procedures for clearing roots and staining parasitic and vesicular-arbuscular mycorrhizal fungi for rapid assessment of infection. T Brit Mycol Soc 55:158-161

Porcel R, Ruiz-Lozano JM (2004) Arbuscular mycorrhizal influence on leaf water potential, solute accumulation, and oxidative stress in soybean plants subjected to drought stress. J Exp Bot 55:1743-1750

Redman RS, Dunigan DD, Rodriguez RJ (2001) Fungal symbiosis from mutualism to parasitism: who controls the outcome, host or invader? New Phytol 151:705-716

Redman RS, Sheehan KB, Stout RG, Rodriguez RJ, Henson JM (2002) Thermotolerance generated by plant/fungal symbiosis. Science 298: $1581-1581$

Regvar M, Likar M, Piltaver A, Kugonič N, Smith JE (2010) Fungal community structure under goat willows (Salix caprea L.) growing at metal polluted site: the potential of screening in a model phytostabilisation study. Plant Soil 330:345-356

Reininger V, Schlegel M (2016) Analysis of the Phialocephala subalpine transcriptome during colonization of its host plant Picea abies. PLoS One 11:1-16

Rozpądek P, Wezowicz K, Stojakowska A, Malarz J, Surówka E, Sobczyk Ł, Anielska T, Ważny R, Miszalski Z, Turnau K (2014) Mycorrhizal fungi modulate phytochemical production and antioxidant activity of Cichorium intybus L. (Asteraceae) under metal toxicity. Chemosphere 112:217-224

Rozpądek P, Wężowicz K, Nosek M, Ważny R, Tokarz K, Lembicz M, Miszalski Z, Turnau K (2015) The fungal endophyte Epichloe typhina improves photosynthesis efficiency of its host orchard grass (Dactylis glomerata). Planta 242:1025-1035

Ruytinx J, Martino E, Rozpądek P, Daghino S, Turnau K, Colpaert J, Perotto S (2016) Homeostasis of trace elements in mycorrhizal fungi. In: Martin F (ed) Molecular mycorrhizal symbiosis. John Wiley \& Sons, Inc., Hoboken, pp 276-298

Santos JM, Vrandečić K, Ćosić J, Duvnjak T, Phillips AJL (2011) Resolving the Diaporthe species occurring on soybean in Croatia. Persoonia 27:9-19

Scervino JM, Gottlieb A, Silvani VA, Pérgola M, Fernández L, Godeas AM (2009) Exudates of dark septate endophyte (DSE) modulate the development of the arbuscular mycorrhizal fungus (AMF) Gigaspora rosea. Soil Biol Biochem 41:1753-1756

Scheper RWA, Crane DC, Whisson DL, Scott ES (2000) The Diaporthe teleomorph of Phomopsis Taxon 1 on grapevine. Mycol Res 104: 226-231
Schulz B, Boyle C (2005) The endophytic continuum. Mycol Res 109: $661-686$

Sebastianes FLS, Lacava PT, Fávaro LCL, Rodrigues MBC, Araújo WL, Azevedo JL, Pizzirani-Kleineret AA (2011) Genetic transformation of Diaporthe phaseolorum, an endophytic fungus found in mangrove forests, mediated by Agrobacterium tumefaciens. Curr Genet 58:21-33

Seddas-Dozolme PM, Arnould C, Tollot M, Kuznetsova E, GianinazziPearson V (2010) Expression profiling of fungal genes during arbuscular mycorrhiza symbiosis establishment using direct fluorescent in situ RT-PCR. In: Sharon A (ed) Molecular and cell biology methods for fungi. Humana Press, New York, pp 137-152

Shen M, Zhao DK, Qiao Q, Liu L, Wang JL, Cao GH, Li T, Zhao ZW (2015) Identification of glutathione S-transferase (GST) genes from a dark septate endophytic fungus (Exophiala pisciphila) and their expression patterns under varied metals stress. PLoS One 10:1-13

Sheoran IS, Singal HR, Singh R (1990) Effect of cadmium and nickel on photosynthesis and the enzymes of the photosynthetic carbon reduction cycle in pigeon pea (Cajanus cajan). Photosynth Res 23:345351

Singh BK, Millard P, Whiteley AS, Murrell JC (2004) Unravelling rhizosphere-microbial interactions: opportunities and limitations. Trends Microbiol 12:386-393

Smith SE, Read DJ (1997a) Vesicular arbuscular mycorrhizas. In: Mycorrhizal symbiosis. Academic Press, San Diego, pp 9-161

Smith SE, Read DJ (1997b) Uptake, translocation and transfer of nutrients in mycorrhizal symbioses. In: Mycorrhizal symbiosis. Academic Press, San Diego, pp 379-408

Smith SE, Read DJ (2008) Arbuscular mycorrhizas. In: Mycorrhizal symbiosis. Academic Press, San Diego, pp 1-188

Soleimani M, Afyuni M, Hajabbas MA, Nourbakhsh F, Sabzalian MR, Christensen JH (2010) Phytoremediation of an aged petroleum contaminated soil using endophyte infected and non-infected grasses. Chemosphere 81:1084-1090

Stefanov KL, Pandev SD, Seizova KA, Tyankova LA, Popov SS (1995) Effect of lead on the lipid metabolism in spinach leaves and thylakoid membranes. Biol Plant 37:251-256

Stone JK, Bacon CW, White JF (2000) An overview of endophytic microbes: endophytism defined. In: Bacon CW, White JF (eds) Microbial endophytes. Marcel Decker Inc., New York, pp 3-29

Strasser RJ, Tsimilli-Michael M, Srivastava A (2004) Analysis of the chlorophyll $a$ fluorescence transient. In: Papageorgiou GC, Govindjee (eds) Chlorophyll $a$ fluorescence. Springer, Houten, pp 321-362

Sun X, Guo LD, Hyde KD (2011) Community composition of endophytic fungi in Acer truncatum and their role in decomposition. Fungal Divers 47:85-95

Suryanarayanan TS, Murali TS, Venkatesan G (2002) Occurrence and distribution of fungal endophytes in tropical forests across a rainfall gradient. Can J Bot 80:818-826

Tripathy BC, Mohanty P (1980) Zinc-inhibited electron transport of photosynthesis in isolated barley chloroplasts. Plant Physiol 66:1174 1178

Trouvelot A, Kough JL, Gianinazzi-Pearson V (1986) Mesure du taux de mycorhization VA d'un système radiculaire. Recherche de methods d'estimation ayant une signification fonctionnelle. In: GianinazziPearson V, Gianinazzi S (eds) Physiological and Genetical aspects of mycorrhizae. INRA-Press, Paris, pp 217-221

Turnau K, Jurkiewicz A, Lingua G, Barea JM, Gianinazzi-Pearson V (2005) Role of arbuscular mycorrhiza and associated microorganisms in phytoremediation of heavy metal-polluted sites. In: MNV P, Sajwan KS, Naidu R (eds) Trace elements in the environment. Biogeochemistry, biotechnology, and bioremediation. CRC Taylor \& Francis, Boca Raton, London, New York, pp 235-252

Turnau K, Orłowska E, Ryszka P, Zubek S, Anielska T, Gawroński S, Jurkiewicz A (2006) Role of mycorrhizal fungi in phytoremediation 
and toxicity monitoring of heavy metal rich industrial wastes in Southern Poland. In: Twardowska I, Allen HE, Häggblom MM, Stefaniak S (eds) Soil and water pollution monitoring, protection and remediation. Springer, Houten, pp 533-551

Van der Putten WH, Vet LE, Harvey JA, Wäckers FL (2001) Linking above- and belowground multitrophic interactions of plants, herbivores, pathogens, and their antagonists. Trends Ecol Evol 16:547554

Villiers F, Ducruix C, Hugouvieux V, Jarno N, Ezan E, Garin J, Junot C, Bourguignon $\mathrm{J}$ (2011) Investigating the plant response to cadmium exposure by proteomic and metabolomic approaches. Proteomics 11:1650-1663

Vodnik D, Jentschke G, Fritz E, Gogala N, Godbold DL (1999) Rootapplied cytokinin reduces lead uptake and affects its distribution in Norway spruce seedlings. Physiol Plantarum 106:75-81

Waller F, Achatz B, Baltruschat H, Fodor J, Becker K, Fischer M, Kogel KH (2005) The endophytic fungus Piriformospora indica reprograms barley to salt-stress tolerance, disease resistance, and higher yield. Proc Natl Acad Sci U S A 102:13386-13391
Wang XN, Huang WY, Du JC, Li CY, Liu JK (2014) Chemical constituents from the fruiting bodies of Xylaria euglossa Fr. and its chemotaxonomic study. Biochem Syst Ecol 54:157-159

Weyens N, van der Lelie D, Taghavi S, Vangronsveld J (2009) Phytoremediation: plant-endophyte partnerships take the challenge. Curr Opin Biotech 20:248-254

Wężowicz K, Rozpądek P, Turnau K (2014) The diversity of endophytic fungi in Verbascum lychnitis from industrial areas. Symbiosis 64: $139-147$

Zhang HW, Song YC, Tan RX (2006) Biology and chemistry of endophytes. Nat Prod Rep 23:753-771

Zhang Y, Zhang Y, Liu M, Shi X, Zhao Z (2008) Dark septate endophyte (DSE) fungi isolated from metal polluted soils: their taxonomic position, tolerance, and accumulation of heavy metals in vitro. $\mathrm{J}$ Microbiol 46:624-632

Zhao D, Li T, Shen M, Wang J, Zhao Z (2015) Diverse strategies conferring extreme cadmium $(\mathrm{Cd})$ tolerance in the dark septate endophyte (DSE) Exophiala pisciphila: evidence from RNA-seq data. Microbiol Res 170:27-35 